\title{
Renforcer l'efficacité de la commande publique
}

\section{Les notes du conseil d'analyse économique, n²2, avril 2015}

\section{$\mathbf{L}$} a commande publique, terme générique qui regroupe les marchés publics, les délégations de service public et les contrats de partenariat, représente aujourd'hui des montants considérables, évalués à presque $15 \%$ du PIB. Si la commande publique doit viser la meilleure performance possible en termes de coûts et de services, elle est régulièrement montrée du doigt pour son inefficacité. De fait, les gains liés à sa meilleure gestion pourraient être substantiels. L'objet de cette Note est de réfléchir aux moyens d'action possibles, dans le cadre de changements réglementaires en cours au niveau européen, pour renforcer l'efficacité de la commande publique.

Les contrats par lesquels s'établit la commande publique sont sujets aux asymétries d'information (l'entreprise connaît mieux ses coûts et l'environnement économique que la partie publique) et à l'incomplétude contractuelle (il est impossible de prévoir tous les événements pouvant survenir durant l'exécution du contrat). Dès lors, l'analyse économique recommande d'utiliser autant que possible les forces concurrentielles dans la phase de sélection des partenaires et de mettre en place des mécanismes incitatifs pour constituer de véritables engagements des parties.

Les nouvelles directives européennes concernant les marchés publics et les concessions, votées en 2014 et qui devront être transposées d'ici 2016, vont conférer à la puissance publique davantage de flexibilité pour négocier avec les entreprises tant dans la phase de sélection que dans la phase d'exécution (renégociations). Cette évolution nous paraît positive et économiquement justi- fiée. Toutefois, il est indispensable de l'accompagner de modalités précises d'encadrement, actuellement non prévues par les directives. Nos recommandations s'articulent autour de trois axes essentiels que sont la transparence, la concurrence et les compétences.

La procédure de négociation doit s'accompagner d'une information transparente avant et après négociation. En phase d'exécution, les avenants aux contrats les plus importants doivent pouvoir être contestés sans pour autant paralyser le processus en facilitant la multiplication des recours futiles. Nous formulons plusieurs recommandations en faveur d'une transparence plus grande de la commande publique.

Pour accroitre la concurrence au moment des appels d'offres, il serait utile de limiter le nombre de plates-formes électroniques d'information et de les faire converger vers un modèle standardisé performant. Dans le même temps, il est aussi souhaitable de simplifier les procédures, de renforcer la professionnalisation des acheteurs publics et de concentrer les achats les plus standards pour bénéficier d'économies d'échelle et cumuler l'expérience des acheteurs publics.

Enfin, pour les projets de grande envergure, nous recommandons de procéder à une évaluation préalable comparative afin de déterminer quel est l'outil de la commande publique le plus adéquat pour répondre aux besoins des autorités publiques. L'agence en charge de cette évaluation préalable réaliserait aussi les évaluations a posteriori pour en tirer des enseignements sur les différents outils et procédures.

b Toulouse School of Economics (TSE) et Institute for Advanced Study in Toulouse (IAST), membre du CAE. 
Faire ou faire faire ? Telle est la première question que doit se poser une administration publique lorsqu'elle souhaite mettre à disposition une nouvelle infrastructure ou un service public. Une fois qu'elle a décidé d'externaliser la tâche (faire faire), la puissance publique se trouve confrontée à un deuxième choix : celui du mode d'externalisation - marché public, délégation de service public ou contrat de partenariat, ensemble que nous désignons ici sous le terme générique de commande publique (voir infra). Enfin, lorsqu'un type d'externalisation est sélectionné, il reste à définir le mode de mise en concurrence des entreprises candidates, le design du contrat et son suivi aux différentes étapes.

Les travaux de recherche existants sur la commande publique suggèrent que l'externalisation a des effets mitigés en termes de qualité de service ou de coûts ${ }^{1}$. Toutefois, ce n'est pas tant la décision d'externaliser que le mode d'externalisation et surtout sa mise en œuvre qui pose problème. Le partage du risque inscrit dans le contrat est une question centrale. Cette Note explore les moyens d'améliorer l'efficacité de la commande publique une fois que la décision d'avoir recours à un partenaire extérieur a été prise. Les sommes en jeu, bien que difficiles à bien cerner, sont considérables. Les achats des administrations publiques représentaient en 2011 environ 15 \% du PIB en France comme au Royaume-Uni et en Allemagne ${ }^{2}$.

\section{Le partage du risque dans les contrats publics:l'apport del'analyse économique}

Le partage du risque entre la personne publique et l'entreprise doit avant tout tenir compte de l'asymétrie d'information entre ces deux acteurs. L'entreprise a naturellement une meilleure connaissance de la technologie disponible, des coûts d'approvisionnement, de la demande pour les produits et services concernés par la commande publique. Par ailleurs, les coûts et la demande ne sont pas indépendants de ses décisions en matière de gestion des ressources humaines, de choix de capacités de production, de recherche et développement, de qualité de service, de gestion des risques, etc. Pour faire face à ces asymétries d'information, il peut être utile, par exemple, d'étalonner les performances de l'entreprise sur celles d'entreprises similaires opérant sur des marchés différents, ou bien de mettre aux enchères une concession de service public pour révéler la plus performante.

Le partage du risque entre les contractants est central dans les situations d'asymétrie d'information : il détermine à la fois le niveau de responsabilisation de l'entreprise et son profit potentiel. Considérons le risque de coût. Deux schémas contractuels (ainsi que des schémas intermédiaires) en termes de partage des coûts sont envisageables ${ }^{3}$ :

- un remboursement de l'entreprise à hauteur de ses coûts assorti d'un paiement prédéfini : contrat à coûts remboursés (cost-plus contract) pour les services non marchands, et contrat de régulation indexant les prix pour l'usager sur le niveau des coûts réalisés (cost-of-service regulation) pour les services marchands. Ce premier schéma déresponsabilise l'entreprise mais limite ses profits potentiels ; - un contrat " à prix fixe " (fixed price contract) dans le cadre de projets publics non marchands, ou « à prix plafonné " (price cap regulation), c'est-à-dire non indexé sur le coût effectif de la production pour les services marchands. Ce second schéma octroie une somme fixe à l'entreprise, quels que soient ses coûts effectifs et quel que soit le niveau de la demande. II suscite davantage d'effort de maîtrise des coûts de la part de l'entreprise, mais lui laisse un profit substantiel lorsque par hasard ses coûts sont particulièrement bas (ou la demande est particulièrement élevée), indépendamment de tout effort de sa part.

La responsabilisation de l'entreprise peut porter sur de nombreuses dimensions : le coût de construction de l'ouvrage, son coût d'exploitation, la demande... Dans le cadre d'un projet d'infrastructure, un moyen de responsabiliser l'entreprise est de lier les tâches de conception/construction à l'exploitation ultérieure : l'entreprise n'a alors pas intérêt à trop serrer les coûts sur la première partie du contrat si cela doit se payer en maintenance durant l'exploitation de l'infrastructure. II peut cependant être difficile pour le donneur d'ordres de mettre en place des contrats de long terme, qui supposent une bonne visibilité sur l'environnement économique, mais aussi sur la capacité de l'entreprise à remplir sa mission sans renégociation a posteriori.

\section{Les trois outils de la commande publique}

\section{Les marchés publics}

Les marchés publics, qui répondent à des besoins en matière de travaux, de fournitures ou de services, ont représenté en France environ 72 milliards d'euros hors taxes (HT) en 2013 (pour 96500 contrats) d'après l'Observatoire économique de l'achat public (OEAP). Toutefois, les données de l'OEAP ne sont pas exhaustives du fait du seuil de déclaration de 90000 euros et de l'absence de contrôle et de sanction en cas de défaut de

Les auteurs remercient les membres du CAE, Salim Bensmail, Frédéric Bobay, Pierre-Emeric Chabanne, Jean-Paul Ducharne, Michel Grevoul, Annick Juillet, Jean Maïa, Frédéric Marty, Robert Stakowski et Maud de Vautibault. Les auteurs remercient également Jean Beuve, conseiller scientifique au CAE, pour son aide précieuse.

${ }^{1}$ Australian Industry Commission (1996) : Competitive Tendering and Contracting by Public Sector Agencies, Australian Government Publishing Service, Rapport $n^{\circ} 48$.

${ }^{2}$ Selon la définition retenue par l'OCDE, les achats publics recouvrent les achats intermédiaires des administrations publiques, les dépenses d'investissement et les transferts sociaux en nature via des services marchands (hors Sécurité sociale). D'autres mesures sont possibles, à partir de la comptabilité nationale ou bien des recensements de contrats. Les écarts entre les différentes mesures peuvent être considérables en raison de différences de champs, de conventions comptables ou du fait d'une collecte d'information très parcellaire sur les contrats.

${ }^{3}$ Laffont J-J. et J. Tirole (1993) : A Theory of Incentives in Procurement and Regulation, MIT Press. 
déclaration. En outre, les dépenses engagées par les opérateurs de l'État et les entreprises à capitaux publics ne sont pas comptabilisées par l'OEAP, pas plus que celles engagées par les entreprises titulaires de délégations de services publics (par exemple, les sociétés autoroutières ou les sociétés d'économie mixte). Le total des marchés publics en France est ainsi estimé aux alentours de 200 milliards d'euros par an, soit $10 \%$ du PIB4 .

\section{Les délégations de service public}

Les délégations de service public (DSP) regroupent l'ensemble des contrats (essentiellement les contrats d'affermage et de concession) par lesquels une personne morale de droit public confie la gestion d'un service public dont elle a la responsabilité à un délégataire public ou privé dont la rémunération est substantiellement liée au résultat d'exploitation du service. Les DSP sont présentes dans les secteurs de la restauration collective, de l'eau et de l'assainissement, du chauffage urbain, des transports, des infrastructures sportives, etc. II n'existe malheureusement pas d'observatoire des DSP en France permettant de recenser les appels d'offres et les contrats signés et ainsi d'évaluer leur poids dans l'économie française. On estime que les DSP en France représentent en valeur, pour les seuls opérateurs ${ }^{5}$, un volume d'affaires de plus de 100 milliards d'euros par an, soit environ $5 \%$ du PIB (dont environ la moitié pour les transports) ${ }^{6}$.

\section{Les contrats de partenariat}

Lancé en juin 2004, le contrat de partenariat (ou Partenariat Public-Privé, PPP) permet à une personne publique de confier à une entreprise une mission globale, dans le cadre d'un contrat de longue durée, contre un paiement effectué par la personne publique et étalé dans le temps. Il est utilisé pour les grands projets de construction (établissements scolaires, gares), d'équipement urbain (éclairage public, voirie) ou encore d'équipement sportif ou culturel (théâtres, stades, piscines). La création du contrat de partenariat a eu notamment pour objectif de rattraper le retard pris en France par rapport à d'autres pays comme le Royaume-Uni, qui dispose depuis le début des années 1990 de ce type d'outil. Les contrats de partenariat se sont beaucoup développés entre 2005 et 2012, date à laquelle ils ont représenté jusqu'à $5 \%$ de l'investissement public en France tout en restant très en deçà des deux autres outils de la commande publique (environ 0,2 \% du PIB).

\section{Les trois outils face au partage du risque}

Les trois outils de la commande publique définis plus haut présentent un partage des risques différent entre la puissance publique et l'entreprise réalisant le contrat (voir tableau). Ainsi, la DSP suppose un paiement non seulement différé mais réalisé par l'usager et non par la puissance publique. Le risque portant sur la demande est supporté par l'entreprise délégataire. Dans le cas des contrats de partenariat, le paiement est différé mais toujours réalisé par la puissance publique qui supporte alors le risque de demande.

Ces trois formes de contractualisation peuvent elles-mêmes être combinées, de sorte qu'il existe en réalité un continuum de contrats possibles. Par exemple, pour tenir compte du risque de trafic et ne pas le transférer totalement au délégataire, suivant en cela la recommandation de certains économistes ${ }^{7}$, des contrats de concession (DSP) prévoient une durée elle-même fonction de

\section{Le transfert de risque dans les différents outils de la commande publique ${ }^{a}$}

\begin{tabular}{|c|c|c|c|}
\hline & $\begin{array}{l}\text { Marchés } \\
\text { publics }\end{array}$ & $\begin{array}{c}\text { Délégations } \\
\text { de service public }\end{array}$ & $\begin{array}{l}\text { Contrats de partenariat } \\
\text { (PPP) }\end{array}$ \\
\hline Paiement différé & Non & $\begin{array}{c}\text { Oui } \\
\text { généralement par l'usager }\end{array}$ & $\begin{array}{c}\text { Oui } \\
\text { par la puissance publique }\end{array}$ \\
\hline Transfert du risque de production (du service ou de l'infrastructure) & Oui & Oui & Oui \\
\hline Transfert du risque lié à la demande & Non & Oui & Non, ou peu \\
\hline Transfert du risque lié au coût d'exploitation & $\begin{array}{l}\text { Oui } \\
\text { dans les marchés de service }\end{array}$ & Oui & $\begin{array}{c}\text { Oui } \\
\text { partiellement }\end{array}$ \\
\hline Contrat global & Non, sauf CREM ${ }^{b}$ & Oui & Oui \\
\hline Maîtrise d'ouvrage & Publique & Privée & Privée \\
\hline Durée du contrat & Court-moyen terme & Moyen-long terme & Long terme \\
\hline \multicolumn{4}{|c|}{$\begin{array}{l}\text { Notes: }{ }^{a} \text { Par " transfert du risque ", nous entendons un transfert vers l'entreprise contractante, c'est-à-dire sa responsabilisation en la matière (coût } \\
\text { ou demande); b Les CREM sont des marchés publics globaux par lesquels une personne publique peut confier à un titulaire unique une mission globale. } \\
\text { Celle-ci peut porter sur la conception et la construction d'ouvrages (marchés de conception-réalisation) ou bien sur la conception et/ou la construction, } \\
\text { l'aménagement, l'entretien et la maintenance (CREM/REM). Ils restent soumis, à la différence des contrats de partenariat, aux règles relatives à la } \\
\text { maîtrise d'ouvrage publique. Ajoutés en } 2011 \text { à la boîte à outils du Code des marchés publics, I'utilisation des CREM et des REM (art. 73) est restreinte } \\
\text { réglementairement et assez faible à ce jour. } \\
\text { Source : Auteurs. }\end{array}$} \\
\hline
\end{tabular}

\footnotetext{
${ }^{4}$ Voir, par exemple, le site de la médiation des marchés publics : www.economie.gouv.fr/mediation-des-marches-publics/guide-osez-commande-publique ${ }^{5}$ Le prix payé par les consommateurs ne va pas toujours intégralement aux opérateurs privés mais peut aller aux collectivités lorsqu'elles financent une partie des investissements.

${ }^{6}$ Institut de la gestion déléguée (IGD) (2011): Aspects économiques et comptables des investissements dans les PPP.

${ }^{7}$ Engel E., R. Fischer et A. Galetovic (2001) : « Least-Present-Value-of-Revenue Auctions and Highway Franchising », Journal of Political Economy, vol. 109, $n^{\circ}$ 5, octobre, pp. 993-1020.
} 
la profitabilité : passé un certain seul de profitabilité, le contrat s'arrête. C'est, par exemple, le contrat du viaduc de Millau signé pour une durée de 78 ans mais qui prévoit une fin anticipée après 2044 dès lors que le chiffre d'affaires réel cumulé actualisé dépassera trois cent soixante-quinze millions d'euros. La durée du contrat s'adapte donc au trafic réalisé. Le risque lié à la demande n'est alors plus entièrement transféré à l'entreprise.

\section{La gouvernance des contrats publics : constats et évolutions à venir}

Les contrats sont généralement conclus à l'issue d'un processus concurrentiel. Ils induisent un enjeu de gouvernance et de compétence dans toutes les phases du contrat : sélection, suivi de l'exécution du contrat, clôture. La difficulté pour la puissance publique est qu'elle ne peut observer directement les performances de l'entreprise (asymétrie d'information), mais aussi qu'elle ne peut prévoir tous les aléas auxquels sera soumis le contrat durant sa réalisation.

\section{Négociation et renégociation des contrats}

Les difficultés commencent au moment de la sélection des entreprises, généralement par appel d'offres : risque de collusion lorsque les marchés sont concentrés ${ }^{8}$; risque de « malédiction du vainqueur " (lorsque la meilleure offre émane du partenaire le plus optimiste et non du plus efficace) ; risque de recevoir des offres excessivement agressives afin d'être retenu, avec l'idée de renégocier ensuite le contrat ; risque de corruption?.

La phase de sélection du partenaire est d'autant plus difficile que la partie adjudicatrice ne peut pas toujours se limiter à un appel d'offres au moins-disant en prix, sans négociation voire sans « dialogue compétitif » (cf. encadré). Comme l'Allemagne, la France accorde une place relativement importante aux appels d'offres avec négociation (30 \% des marchés publics en valeur si l'on additionne les procédures négociées et le dialogue compétitif), l'appel d'offres ouvert sans négociation demeurant cependant la règle ( $c f$. graphique). De son côté, le Royaume-Uni a davantage recours aux appels d'offres restreints.

Constat 1. Les procédures de sélection négociées sont peu utilisées en Europe.

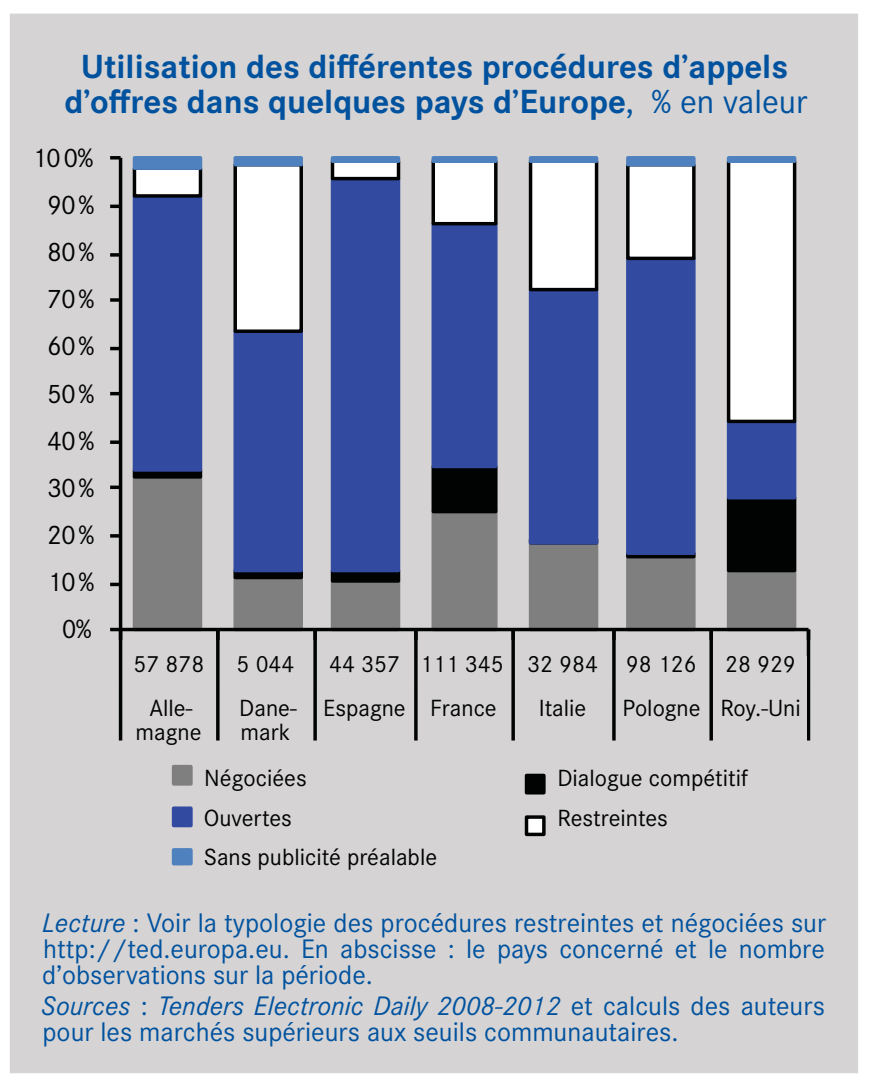

La sélection des entreprises est rendue plus difficile encore par la fréquente renégociation des contrats après leur signature, une pratique particulièrement répandue pour les DSP (les taux de renégociation varient entre 40 et $92 \%$ selon les secteurs et les pays étudiés ${ }^{10}$ ). Les renégociations fragilisent les appels d'offres en favorisant les comportements opportunistes (enchères agressives où l'opérateur fait volontairement une offre à faible prix en anticipant le fait qu'il renégociera le contrat durant la phase d'exécution). Le mécanisme d'appel d'offres ne conduit donc plus forcément à sélectionner le meilleur candidat (le moins-disant ou le mieux-disant) mais celui qui a le plus confiance dans son pouvoir de renégociation ${ }^{11}$. II est aussi très difficile dans ces conditions de mettre en place un contrat réellement incitatif. Ainsi, la renégociation des contrats tend à réduire, voire à faire disparaître, les bienfaits des procédures concurrentielles d'attribution ${ }^{12}$.

Constat 2. Une part importante des contrats publics avec des partenaires privés est ultérieurement renégociée.

\footnotetext{
${ }^{8}$ Au sein des marchés publics, l'exemple des lycées d'Île-de-France fournit une bonne illustration des stratégies que les entreprises peuvent mettre en place pour éliminer la concurrence lors des appels d'offres (voir décision de 2007 n 07-D-15 de l’Autorité de la concurrence) ; pour les DSP, voir, par exemple, le cas du transport public urbain de voyageurs (décision de $2005 \mathrm{n}^{\circ}$ 05-D-38 de l'Autorité de la concurrence).

${ }^{9}$ Dans son rapport anticorruption (COM(2014) 38 final), I'Union européenne notait que la corruption à elle seule coûte chaque année 120 milliards d'euros à l'économie européenne et que les marchés publics sont particulièrement touchés. La corruption pourraient augmenter les coûts des achats publics de 20 à $25 \%$ et, dans certains cas plus limités, les augmenter de 50 \%. L'OCDE note que "l les intérêts financiers en jeu et l'interaction étroite entre les secteurs public et privé font de la passation des marchés publics une zone de risque majeur [...] ॥, p. 21.

${ }^{10}$ Estache A. et S. Saussier (2014) : "Public-Private Partnerships and Efficiency: A Short Assessment », CESifo DICE Report, ${ }^{\circ} 3 / 2014, \mathrm{pp} .8-13$.

${ }^{11}$ Voir Athias L. et A. Nuñez (2008) : « Winner’s Curse in Toll Road Concessions », Economics Letters, vol. 101, n 3, décembre, pp. $172-174$.

${ }^{12}$ Voir Guasch J-L. (2004) : Granting and Renegotiating Infrastructure Concession: Doing It Right, The World Bank. Cette conclusion est obtenue à partir de l'analyse de plus de 1000 contrats de concession signés en Amérique latine.
} 
Toutefois, les renégociations sont utiles lorsqu'elles permettent au contrat de s'adapter à des situations nouvelles, notamment pour les contrats complexes et de longue durée. L'enjeu est donc de favoriser les adaptations bénéfiques tout en luttant contre les renégociations opportunistes. Ceci passe par la transparence et par la compétence de la partie publique.

\section{Transparence et compétence}

Une étude menée par l'OCDE ${ }^{13}$ note les insuffisances des marchés publics en matière de transparence. En particulier, l'information sur les avenants aux contrats et le suivi des dépenses dans les marchés publics est difficile d'accès dans un grand nombre de pays. Ce constat vaut pour tous les outils de la commande publique.

Or, la transparence est clé à tous les stades de la commande publique pour assurer la liberté d'accès (possibilité d'avoir librement connaissance des besoins émis par la personne publique) et l'égalité de traitement (interdiction de toute discrimination qui concerne tous les stades de la procédure). La renégociation des contrats après leur signature et leur exécution doivent aussi pouvoir être suivis par les contribuables qui les financent. Si la transparence a un coût immédiat, tant pour les pouvoirs publics que pour les opérateurs privés, elle est essentielle au respect des principes qui sont à la base du système de commande publique et est indispensable pour la mesure de sa performance. L'enjeu est alors de permettre une diffusion large et rapide de l'information concernant les phases d'attribution et d'exécution de tous les contrats liés à la commande publique.

Constat 3. Les données manquent pour suivre l'évolution de la commande publique et en analyser les performances. Le contrôle ex ante et ex post des contrats s'en trouve de fait limité.

Une gouvernance fine des contrats publics nécessite des acteurs informés, indépendants, mais aussi compétents pour faire face à des situations parfois complexes. Dans son rapport portant sur l'intégrité dans les marchés publics, l'OCDE note le manque et l'inadéquation des compétences des agents responsables de la commande publique. Les fonctions d'acheteur et de gestionnaire de projets requièrent non seulement des compétences en droit mais aussi en analyse économique et gestion financière. Le besoin de ces multiples compétences est essentiel, aussi bien dans la phase d'attribution (pour la planification, la budgétisation, les choix contractuels ou la gestion des risques) que dans la phase

\section{Les procédures d'attribution formalisées des marchés publics (au-dessus des seuils communautaires)}

Un appel d'offres est une procédure de marchés publics par laquelle la personne publique se prononce en faveur de l'offre économique la plus avantageuse. La sélection se fait sans aucune négociation et sur des critères précis, objectifs et connus du candidat. L'appel d'offres est dit ouvert lorsque toute entreprise peut remettre une offre.

Dans un appel d'offres restreint, seuls les candidats présélectionnés sur la base de leurs capacités (chiffre d'affaires, moyens humains, etc.) sont invités à déposer une offre.

Dans la procédure négociée, après la remise des offres, le pouvoir adjudicateur est amené à négocier les offres sur leurs dimensions techniques et/ou financières, avec un ou plusieurs candidats. La négociation est toujours possible dans les secteurs réseaux; dans les autres secteurs, elle est autorisée au-dessus d'un certain seuil défini au niveau européen et dans des cas exceptionnels.

Si la personne publique n'est pas en mesure de réunir seule les moyens techniques pour exprimer son besoin ou pour établir le montage juridique ou financier de son projet, elle peut avoir recours à une procédure dite de dialogue compétitif. Dans ce cas, elle sélectionne un certain nombre de candidats et procède à plusieurs phases de dialogue afin de mieux cerner ses besoins et les solutions techniques existantes avec eux avant de pouvoir lancer l'appel d'offres.

a Les directives ne s'appliquent que pour les marchés au-dessus des seuils communautaires : pour les marchés de travaux, la procédure par appel d'offres est appliquée dès 5,186 millions d'euros HT sans distinction d'organismes ou de secteurs. Pour les marchés de services et de fournitures, le seuil est de 207000 euros HT pour les collectivités territoriales, de 134000 euros HT pour les structures étatiques et de 414000 euros HT pour les secteurs réseaux Au-dessous de ces seuils, l'acheteur est libre d'organiser sa procédure comme il l'entend, dans le respect des principes constitutionnels de liberté d'accès à la commande publique, d'égalité de traitement des candidats et de transparence des procédures.

d'exécution (pour se prémunir contre des retards injustifiés ou des surcoûts ou le risque de voir l'entreprise contractante accentuer ses avantages si la partie publique se désintéresse de la gestion quotidienne de la délégation). Or la gestion de la commande publique est souvent confiée à des personnes titulaires d'une formation exclusivement juridique. En outre, les changements fréquents d'affectation de ces personnels limitent l'apprentissage " sur le tas » et la « mémoire institutionnelle ». Cela réduit la capacité de la puissance publique à superviser et à contrôler efficacement des contrats qui durent parfois plusieurs décennies. Il existe donc une double asymétrie en matière d'expertise et d'information qui favorise la partie privée et qui encourage les renégociations opportunistes visant, pour l'entreprise, à s'approprier une plus grande partie de la rente. 
Constat 4. Les compétences et les incitations de l'acheteur public sont trop limitées dans un environnement où les asymétries d'information sont omniprésentes et où les détails contractuels sont importants.

\section{De nouvelles directives européennes favorables à la négociation, à la simplification et à la renégociation}

Le nouveau paquet législatif européen sur les marchés publics, qui doit être transposé avant le 18 avril $2016^{14}$, offre une opportunité pour faire évoluer la commande publique en France. Sont concernées les règles de gouvernance de l'ensemble des outils de la commande publique, y compris les contrats de partenariat, notamment dans les phases d'attribution et d'exécution des contrats. La facilité du recours à la négociation est l'un des principaux apports des nouvelles directives marchés publics de 2014. La procédure négociée, désormais appelée " procédure concurrentielle avec négociation " et le dialogue compétitif peuvent désormais être utilisés lorsque les besoins du pouvoir adjudicateur ne peuvent être satisfaits par des solutions immédiatement disponibles, lorsque les prestations portent sur de la conception ou des solutions innovantes, ou encore lorsque le marché est " complexe ॥. Le recours à l'appel d'offres simple ne sera imposé que pour l'achat de produits, services ou travaux standardisés. Cet assouplissement du recours à la procédure négociée rapproche la directive "secteurs classiques 》 de celle relative aux secteurs de réseaux. Par ailleurs, la procédure négociée devient le schéma d'attribution ordinaire des contrats de concession.

Afin d'alléger les charges administratives qui pèsent sur les entreprises qui candidatent aux marchés publics, les directives généralisent le système de la déclaration sur l'honneur. Cette déclaration s'appuiera sur un formulaire européen uniformisé - le Document unique de marché européen (DUME), dont le modèle a été présenté par la Commission en janvier 2015. Seul le candidat auquel il est envisagé d'attribuer le marché devrait être contraint de fournir les documents justifiant l'exactitude des informations figurant dans le DUME. Par ailleurs, la dématérialisation des échanges d'information dans le cadre des procédures sera obligatoire à compter du 18 octobre 2018.

Jusqu'à présent, les directives sur les marchés publics étaient cantonnées aux seules règles de passation. Les nouvelles directives énoncent désormais les hypothèses dans lesquelles les marchés peuvent être modifiés en cours d'exécution. Sont autorisées les modifications d'un montant infé- rieur à $10 \%$ de la valeur initiale du marché pour les fournitures et services et $15 \%$ pour les travaux, ainsi celles qui, quel que soit leur montant, ne sont pas substantielles ou ont été prévues dans le contrat sous forme de clauses de révision de prix ou d'options claires. Des avenants peuvent en outre être conclus en cas d'événements imprévus ou lorsque des prestations supplémentaires sont devenues nécessaires et qu'un changement de contractant est impossible ou présenterait un inconvénient majeur, pour autant que la modification ne modifie pas la nature globale du contrat et qu'elle n'entraîne pas une augmentation de plus de 50 \% du montant du marché. En cas de modifications successives, la directive précise que ce seuil de $50 \%$ s'applique à chaque modification et non à leur montant cumulé. Les concessions peuvent être elles aussi très largement modifiées dans les mêmes circonstances et dans les mêmes proportions que les marchés publics.

\begin{abstract}
Constat 5. La transposition à venir des directives européennes va assouplir les règles de passation et d'exécution des contrats en développant la négociation dans la phase d'attribution et en facilitant les renégociations lors de la phase d'exécution.
\end{abstract}

\section{Améliorer la gouvernance des contrats publics}

Les nouvelles dispositions liées aux directives marchés publics et concessions marquent un mouvement vers une nouvelle vision de la gestion des services publics accordant davantage de liberté aux autorités adjudicatrices. Toutefois, pour tirer pleinement le bénéfice de cette évolution en termes d'efficacité de la commande publique, il est nécessaire de progresser simultanément sur trois dimensions complémentaires - transparence, concurrence, compétences - tout en clarifiant les objectifs de la commande publique ${ }^{15}$.

\section{Préciser les objectifs de la commande publique}

Comme on l'a vu plus haut, les montants importants de la commande publique en font un possible levier pour atteindre des objectifs sociaux, environnementaux ou d'innovation, le donneur d'ordres devant alors incorporer ces critères dans la phase de sélection des entreprises. Les nouvelles directives marchés publics et concession laissent aux États le soin de rendre ces objectifs obligatoires ou facultatifs. Sans remettre en cause la légitimité des objectifs poursuivis, il

\footnotetext{
${ }^{14}$ Directives 2014/24/UE relative à la passation des marchés publics (secteurs dits « classiques ॥) et 2014/25/UE relative à la passation de marchés dans les secteurs de l'eau, de l'énergie, des transports et des services postaux (secteurs dits " spéciaux "), cf. Journal officiel de l'Union européenne, 28 mars 2014. Par ailleurs, la directive du Parlement européen et du Conseil sur l'attribution des concessions (qui correspondent aux délégations de service public françaises) a été adoptée par le Parlement européen le 15 janvier 2014 et par le Conseil de l'Union européenne le 11 février 2014 , directive 2014/23/UE.

${ }^{15}$ Les recommandations formulées par la suite ne s'appliquent qu'aux marchés et DSP concernés par les directives européennes, c'est-à-dire dont les montants sont situés au-dessus des seuils communautaires (voir encadré).
} 
faut noter que la puissance publique dispose de moyens plus directs et efficaces (via les taxes et/ou les subventions) pour les atteindre. II est incongru, par exemple, de taxer à peine les émissions de carbone tout en incorporant des critères vagues de soutenabilité environnementale dans les marchés publics.

Passer par la commande publique pour atteindre des objectifs sociaux, environnementaux ou d'innovation est inefficace pour plusieurs raisons. Tout d'abord, une politique corrigeant une défaillance de marché doit être uniforme et globale pour être efficace. À l'inverse, et pour prendre un exemple, incorporer des critères d'émissions de gaz à effet de serre dans une commande publique revient à attribuer une valeur plus élevée à la tonne de carbone que la taxe carbone ou la valeur sur le marché des droits d'émission négociables, ce qui est inefficace pour deux raisons. D'une part, comme pour toute politique induisant une différenciation des prix du carbone, cela augmente le coût total de réalisation de l'objectif environnemental. D'autre part, une entreprise émettant peu se spécialisera dans les marchés publics, où elle aura un avantage concurrentiel, tandis que son homologue plus polluante se spécialisera sur d'autres marchés, publics ou privés, non contraints par cet objectif ; le résultat sera un partage des marchés pas forcément rationnel et peu de réduction des émissions polluantes.

Ensuite, de tels objectifs créent des difficultés de mesure. Le donneur d'ordres, contrairement à l'État, n'est pas forcément équipé pour mesurer la pollution. En outre, les entreprises sont souvent actives sur plusieurs marchés, publics et privés. Dès lors, comment savoir si telle pollution est liée à l'activité concernée ou à une autre (l'entreprise choisira toujours l'allocation qui lui est favorable) ? De plus, la différentiation entre entreprises s'en trouvera accrue, réduisant l'intensité de la concurrence entre elles. Enfin, la prise en compte d'objectifs divers accroît le risque (toujours présent) de favoritisme. Un donneur d'ordres peut, par exemple, mettre une pondération importante sur le contenu en emploi local.

\footnotetext{
Recommandation 1. Reconnaître que l'objectif de la commande publique, quel qu'en soit le montant, est avant tout de satisfaire un besoin identifié en parvenant à la meilleure performance en termes de coûts et de services ou fonctionnalités attendus. Charger la commande publique d'atteindre des objectifs sociaux, environnementaux ou d'innovation est inefficace.
}

\section{Renforcer la concurrence}

\section{Encadrer la négociation lors de la sélection des offres}

Les directives européennes ouvrent une large part à la négociation lors de la sélection des offres. Bien encadrée, cette négociation peut être très bénéfique pour la commande publique :

- pour les opérations complexes, la négociation permet potentiellement un meilleur calibrage des contrats en aidant la partie publique à préciser ses besoins et en faisant émerger des solutions concurrentielles non anticipées, éventuellement innovantes ${ }^{16}$;

- la négociation lors de la phase de sélection réduit la probabilité de voir le contrat renégocié ultérieurement ${ }^{17}$ sans pour autant nécessairement augmenter les prix ${ }^{18}$;

- les entreprises sont amenées à justifier les éléments qui composent leurs offres lors de la phase de négociation, ce qui réduit le risque de collusion et les offres de couverture (les offres volontairement surévaluées destinées à guider le choix de la partie adjudicatrice vers l'entreprise choisie par l'entente).

Au total, il nous semble qu'introduire une phase de négociation entre les contractants est positif. D'un point de vue théorique, les coûts de transaction ex ante que génèrent les procédures négociées permettent d'établir des contrats plus complets, donc plus robustes à la renégociation ${ }^{19}$.

Néanmoins, outre le risque de favoritisme, la procédure négociée présente un écueil potentiel. La collectivité pourrait vouloir l'utiliser pour faire baisser le prix si la phase de négociation ne porte que sur la proposition financière des enchérisseurs. Anticipant ceci, les entreprises enchériront à un niveau plus élevé initialement. Ainsi, la négociation n'a d'intérêt que si elle change les spécificités techniques désirées par la collectivité. Cependant, si la négociation porte sur les dimensions techniques des offres, elle peut favoriser un concurrent en lui permettant d'améliorer son offre initiale. La procédure négociée présente donc un risque de "pillage " des idées des concurrents.

Le droit européen prévoit un principe de traçabilité et de transparence des négociations. II n'en définit cependant pas précisément les modalités. La transposition des directives donne l'opportunité de retenir des mesures assurant un meilleur contrôle de la négociation.

Pour mettre en œuvre le principe de traçabilité et éviter ainsi les écueils de la procédure négociée, nous proposons que la

\footnotetext{
${ }^{16}$ Bajari P., R.S. McMillan et S. Tadelis (2009) : "Auctions versus Negotiations in Procurement: An Empirical Analysis », Journal of Law, Economics, and Organization, vol. 25, $\mathrm{n}^{\circ}$ 2, pp. 372-399.

${ }^{17}$ Guasch J-L., J-J. Laffont et S. Straub (2008) : «Renegotiation of Concession Contracts in Latin America: Evidence from the Water and Transport Sectors ॥, International Journal of Industrial Organization, vol. 26, $\mathrm{n}^{\circ}$ 2, pp. 421-442.

${ }^{18}$ Chever L. et J. Moore (2012) : « Negotiated Procedures Overrated? Evidence from France Questions the Commission's Approach in the Latest Procurement Reforms ", European Procurement and Public Private Partnership Law Review, vol. 4, pp. 228-241.

${ }^{19}$ Voir Tirole J. (2009) : « Cognition and Incomplete Contracts », American Economic Review, vol. 99, n 1, mars, pp. $265-294$.
} 
collectivité soit tenue d'établir deux rapports synthétiques : le premier porterait sur l'analyse des offres avant le début de la négociation; le second porterait sur l'analyse des offres après la clôture des négociations. Ces deux rapports existent déjà dans la procédure "Sapin ॥ qui s'applique aux délégations de service public. Ils seraient généralisés à l'ensemble des procédures négociées. Pour les délégations de service public, la nouveauté serait l'obligation d'indiquer quels ont été les acquis de la négociation, en particulier sur les aspects techniques des offres et le contenu des engagements des candidats. Pour les marchés publics, le travail supplémentaire engendré par ces rapports serait compensé par l'allégement prévisible du rapport justifiant le recours à la négociation.

\begin{abstract}
Recommandation 2. Rendre obligatoire pour la partie publique la fourniture et la publication en ligne de deux rapports synthétiques sur l'analyse des offres avant et après la clôture des négociations.
\end{abstract}

\section{Concurrence et réputation des candidats}

La directive sur les marchés publics offre la possibilité d'exclure une entreprise soumissionnaire en cas de défaillance sur un contrat passé20. Cette disposition est de nature à inciter les entreprises à respecter leurs engagements contractuels et peut éviter les comportements stratégiques visant, par exemple, à remporter un marché par sous-estimation des coûts. Elle est d'autant plus importante pour la partie publique que le contrat est complexe et donc que la probabilité de devoir renégocier est grande ${ }^{21}$. L'intérêt de ce mécanisme a par ailleurs été confirmé par une étude empirique menée sur une grande entreprise italienne de services publics ayant introduit pour ses sous-traitants un système de notation à titre expérimental pour ensuite utiliser un critère de réputation dans les procédures de sélection. L'étude conclut que cela a augmenté la qualité, et que des règles simples de notation de la réputation peuvent éviter le favoritisme et la création de barrières à l'entrée pour de nouveaux entrants sans expérience passée avec le contractant public $^{22}$. Ces résultats, sans pouvoir être généralisés à tous les secteurs et tous les pays, suggèrent que les entreprises valorisent leur réputation et qu'il peut être utile d'en tenir compte lors des procédures de sélection.

Cependant, des efforts devront être fournis pour rendre plus facilement accessible aux pouvoirs adjudicateurs l'information sur la réputation des entreprises et pour rendre objective et vérifiable cette information afin que les collectivités qui l'utiliseraient ne se trouvent pas en difficulté (leur décision d'exclusion pourrait être contestée comme non fondée et restreignant la concurrence). De tels systèmes de partage de l'information existent aux États-Unis ${ }^{23}$ ou encore en Corée du Sud ${ }^{24}$. Sans nécessairement organiser l'exclusion temporaire des entreprises aux appels d'offres comme en Corée du Sud, la centralisation et le partage facilité de l'information sur leurs performances sur les cinq dernières années, par le biais d'une plate-forme nationale dédiée ou d'une extension de la Plate-forme des achats de l'État (PLACE) existante, pourrait renforcer l'efficacité de la commande publique - la possibilité offerte par les nouvelles directives d'exclure temporairement une entreprise en fonction de ses performances passées serait ainsi rendue plus aisée.

\section{Recommandation 3. Centraliser l'infor- mation concernant la performance passée des entreprises afin de faciliter et d'encourager son utilisation lors de la phase d'attribution, pour pouvoir pénaliser sans risque juridique les entreprises peu fiables selon les modalités prévues par la directive.}

\section{La dématérialisation des marchés}

La dématérialisation des marchés (la possibilité de conclure des marchés par voie électronique, soit par l'utilisation de la messagerie électronique, soit par l'emploi d'une plateforme en ligne sur Internet) permet de diffuser plus rapidement et plus largement l'information. La dématérialisation concerne aujourd'hui environ $11 \%$ du montant des marchés en France selon I'OEAP, tandis que la directive européenne prévoit une dématérialisation totale à l'horizon 2018. À côté de cet objectif, une réflexion doit être menée sur le nombre de plates-formes électroniques de publicité et de candidature. Aujourd'hui, de telles plates-formes existent à tous les

\footnotetext{
${ }^{20}$ Les exclusions sont possibles d'après l'article 57 de la directive si « des défaillances importantes ou persistantes de l'opérateur économique ont été constatées lors de l'exécution d'une obligation essentielle qui lui incombait dans le cadre d'un marché public antérieur, d'un marché antérieur passé avec une entité adjudicatrice ou d'une concession antérieure, lorsque ces défaillances ont donné lieu à la résiliation dudit marché ou de la concession, à des dommages et intérêts ou à une autre sanction comparable ".

${ }^{21}$ Tadelis S. (2006) : Incentives and Award Procedures: Competitive Tendering versus Negotiations in Procurement, Handbook of Procurement, Cambridge University Press, pp. 121-139.

${ }^{22}$ Spagnolo G. (2012) : « Reputation, Competition and Entry in Procurement », International Journal of Industrial Organization, vol. $30, \mathrm{n}^{\circ} 3$, pp. $291-296$.

${ }^{23}$ Aux États-Unis, depuis 2010, I'application Internet Federal Awardee Performance and Integrity Information System (FAPIIS) agrège de multiples sources d'informations (dont le Past Performance Information Retrieval, PPIRS, et le Contractor Performance System, CPS) et aide les services fédéraux chargés des achats à sélectionner les fournisseurs en leur donnant des informations pertinentes et actualisées sur les antécédents d'une entreprise sur les cinq dernières années.

${ }^{24}$ Sous l'égide de l'agence publique qui regroupe les achats de l'État et des administrations publiques, les entreprises qui ne respectent pas les délais contractuels sont « blacklistées » pendant trois mois si le retard de livraison observé est inférieur à un mois, pendant six mois pour un retard inférieur à six mois, pendant un an pour un retard supérieur à six mois. En cas de non-respect de la qualité, l'entreprise est sanctionnée pendant un an.
} 
échelons administratifs : communal, départemental, régional et même national avec l'introduction de la PLACE. Alors que la dématérialisation vise à faciliter l'accès à la commande publique, la dispersion de l'information sur une multitude de sites Internet oblige les entreprises à financer des services de recherche des marchés qui pourraient les intéresser et limite ainsi leur participation (notamment celle des PME) aux appels d'offres. En outre, chaque plate-forme comporte ses propres spécificités d'utilisation et les documents demandés ne sont pas toujours les mêmes, ce qui multiplie les coûts pour les entreprises candidates.

L'exemple coréen est instructif de ce point de vue. Le Public Procurement Service - PPS y a développé une plateforme électronique, KONEPS (Korea ON-line E-Procurement System), mise en place en 2002. KONEPS a géré pour plus de 55,5 milliards d'euros de commande publique en 2013 , soit environ $65 \%$ du total de la commande publique du pays. Utilisée par plus de 267000 entreprises et 46000 organisations publiques, la plate-forme améliore la transparence et l'information des parties et a grandement simplifié les procédures pour répondre aux appels d'offres (par exemple, les soumissionnaires n'ont qu'un dossier unique à remplir pour toutes leurs candidatures futures).

\begin{abstract}
Recommandation 4. Mettre en place (ou maintenir) des plates-formes électroniques de publicité et de candidature uniquement au niveau régional et faire remonter toute l'information à une plate-forme nationale. Faire converger les pratiques des différentes plates-formes vers les initiatives régionales les plus performantes.
\end{abstract}

\section{Renforcer la transparence}

L'objectif des propositions qui suivent est d'augmenter la transparence dans la passation et l'exécution des contrats publics. II ne s'agit pas d'améliorer la transparence par principe (quelquefois plus de transparence peut entraîner des effets négatifs en facilitant par exemple les ententes), mais de mieux responsabiliser les décideurs publics.

\section{Transparence et responsabilisation des acheteurs publics}

Une plus grande transparence de la commande publique est la contrepartie indispensable de procédures d'attribution plus flexibles et de la possibilité accrue de renégocier les contrats. Au-delà des évolutions suggérées plus haut, les pouvoirs adjudicateurs devraient être tenus de publier systé- matiquement des données sur la commande publique, particulièrement sur les raisons de leurs choix. En effet, dès lors que c'est l'offre économiquement la plus avantageuse ${ }^{25}$ qui est retenue, en application du ou des critères annoncés dans l'avis d'appel public à la concurrence ou dans le règlement de la consultation, il est utile d'informer les candidats malheureux ainsi que les parties prenantes des raisons qui ont poussé l'autorité publique à retenir une offre plutôt qu'une autre. Le choix est effectué par la Commission d'appel d'offres (CAO) pour les collectivités territoriales. Si aucun texte ne prévoit explicitement de rapport particulier, le ministère de l'Économie a néanmoins mis en ligne un modèle de rapport d'analyse des offres et de procès-verbal de la réunion de la CAO au terme de laquelle elle choisit la meilleure offre. Aujourd'hui, les rapports ne sont communicables que sur demande des entreprises dont l'offre n'a pas été retenue. Un recours auprès de la Commission d'accès aux documents administratifs est nécessaire en cas de refus opposé par la personne publique. Pour augmenter la transparence et responsabiliser les acheteurs publics, nous proposons que ces rapports - qui existent déjà dans la très grande majorité des cas - soient rendus publics. La dématérialisation des marchés et la mise en place de plates-formes centralisant les offres peuvent faciliter la transparence des marchés publics et être le lieu où les rapports seraient publiés.

\begin{abstract}
Recommandation 5. Rendre obligatoire la publication en ligne, en plus des informations légales (procédure, offre retenue, nombre d'enchérisseurs...), du rapport d'analyse des offres.
\end{abstract}

\section{Encadrer et rendre transparentes les renégociations}

Les nouvelles directives européennes reconnaissent le caractère incomplet des contrats de commande publique et laissent une large place à de possibles renégociations dans la phase d'exécution des contrats. Si cette initiative est bienvenue pour s'adapter à des événements imprévus, il est toutefois important que les parties se sentent tenues par le contrat. En l'absence d'engagement, l'une des parties peut perdre le montant d'un investissement spécifique au contrat, la mise en concurrence initiale peut être faussée et le contribuable ou l'usager lésé. II s'agit donc de donner une incitation au donneur d'ordres et aux entreprises contractantes à bien définir la nature du contrat et à bien indexer ce dernier sur les réalisations de variables observables exogènes (comme l'inflation) afin de limiter au maximum la possibilité de renégociation. Pour autant, le risque zéro de renégociation n'existe pas. La renégociation peut d'ailleurs servir à améliorer le contrat initial. Accroître la transparence des procédures de

\footnotetext{
${ }^{25}$ L'offre économiquement la plus avantageuse est celle qui satisfait au mieux l'ensemble des critères d'attribution du marché. Elle dépend de la pondération des critères - subjective - et de la notation de l'offre - elle-aussi en partie subjective - réalisée par l'acheteur sur chacun des critères. Le choix de l'offre économiquement la plus avantageuse s'oppose au choix du moins-disant, fondé sur le seul critère financier et ne laissant ainsi pas de marge discrétionnaire à l'acheteur.
} 
renégociation permettrait de limiter ce problème et assurer ainsi le respect des règles régissant les renégociations.

Une manière simple et peu coûteuse d'augmenter la transparence serait de mettre en place une procédure contentieuse spécifique aux avenants des contrats de la commande publique : une procédure de "référé-avenant ». Cette procédure serait rapide, comme le sont les référés précontractuel ou contractuel ${ }^{26}$. Elle améliorerait la transparence et la responsabilisation des autorités publiques. Le référé-avenant permettrait à tout tiers intéressé (préfet, élus, entreprises, citoyens) de demander l'annulation d'un avenant. Tout nonrespect des règles légales (calcul du seuil, modification de l'objet du contrat, fraude...) ou tout surcoût injustifié serait des motifs invocables. Le délai de recours pourrait être fixé à un mois à compter de la publication d'un « avis d'avenant » sur un support permettant aux tiers intéressés d'avoir connaissance de sa passation (par exemple au Bulletin officiel des annonces des marchés publics) ${ }^{27}$. Les propositions d'avenant seraient simultanément publiées et envoyées électroniquement aux entreprises ayant candidaté, à une liste à définir de tiers intéressés et plus généralement à tout tiers qui en aura fait préalablement la demande. Afin de rendre le recours effectif, la publication devra préciser le montant initial du marché, le montant de l'augmentation et l'objet de l'avenant. Toute personne qui en ferait la demande pourrait obtenir communication de l'avenant et de ses actes détachables. Le délai de recours d'un mois serait, comme en matière de référé précontractuel, un délai de standstill (c'està-dire que l'avenant ne deviendrait exécutoire qu'après l'expiration du délai). En contrepartie, une fois le délai écoulé, l'avenant ne pourrait plus être contesté sur ses aspects purement juridiques ${ }^{28}$.

La procédure de référé-avenant interviendrait en amont, et donc serait plus effective qu'une évaluation a posteriori. Cependant, son utilisation ne va pas de soi. Tout d'abord, il y a un problème de passager clandestin : les gains associés à une bonne décision en référé-avenant sont diffus. Ensuite, les entreprises du secteur ne seront pas toujours disposées à contester le bien-fondé d'une renégociation, car leur gain sera en général minime alors qu'elles risqueraient de se mettre à dos le donneur d'ordres ${ }^{29}$.
En conséquence, la possibilité de soumettre des commentaires amicus curiae au juge lors de la procédure de référéavenant pourrait être utile. On devrait aussi s'interroger sur la possibilité de commenter de façon confidentielle (l'identité du communiquant n'étant pas révélée au donneur d'ordres pour éviter des représailles dans des contrats futurs). II faudrait évidemment éviter qu'à l'inverse un déluge d'informations ne noie le juge. Des documents courts synthétisant l'argument principal (éventuellement complétés par des annexes) pourraient contribuer à cet objectif.

\begin{abstract}
Recommandation 6. Rendre obligatoire la publication d' « avis d'avenant » dès lors que la valeur du contrat est modifiée de plus de $10 \%$ et mettre en place une procédure rapide de référé-avenant ouverte aux parties prenantes. Envisager éventuellement des mécanismes assurant l'anonymat des parties à l'origine de la procédure.
\end{abstract}

\section{Introduire plus de transparence (en parallèle) pour les activités gérées en management public}

Comme on l'a vu, la puissance publique peut aussi choisir de " faire » plutôt que de « faire faire », et de nombreuses collectivités ont par exemple choisi ces dernières années d'internaliser la gestion de l'eau au sein de régies municipales. Pour correctement évaluer les différents outils de la commande publique, il est nécessaire d'étendre les exigences de transparence mentionnées plus haut aux activités gérées directement par la puissance publique lorsque celles-ci pourraient faire l'objet d'un contrat avec un prestataire externe (gestion de l'eau, des cantines, du stationnement...) $)^{30}$.

\section{Recommandation 7. Appliquer aux activités en gestion directe des exigences de transparence, avec des incitations et des sanctions adéquates, afin de permettre une évaluation de ces activités analogue à celles déléguées à un partenaire privé.}

\footnotetext{
${ }^{26}$ Le référé précontractuel ou contractuel est une procédure rapide permettant de demander à une juridiction qu'elle annule totalement ou partiellement une procédure ou un marché. II y en a eu un peu moins de 1100 en 2013 avec un délai moyen de jugement de 21 jours, cf. Conseil d’État (2014) : Le juge administratif et la commande publique, Dossier thématique, juin, p. 4.

${ }^{27}$ Pour les marchés et concessions situés au-dessus des seuils européens, les nouvelles directives « Marchés » et « Concessions » prévoient l'obligation pour la collectivité de publier un « avis de modification » du marché ou de la concession - art. 43 de la directive 2014/23/UE, art. 72 de la directive 2014/24/UE et art. 89 de la directive 2014/25/UE.

${ }^{28}$ Le juge administratif serait habilité à prononcer l'annulation de l'avenant. II conviendrait que la décision intervienne dans un délai réduit, afin de ne pas bloquer les projets qui nécessitent une exécution rapide de l'avenant. La voie unique de recours serait le recours en cassation, à l'instar de ce qui existe pour les référés précontractuel et contractuel.

${ }^{29}$ On peut ici faire l'analogie avec le droit d'éliciter la notification d'actes répréhensibles (whistleblowing) au sein de l'entreprise : les employés et commissaires aux comptes, par exemple, ont plus à perdre qu'à gagner, tandis que les parties les plus incitées à exposer ces actes sont les journalistes et les employés bénéficiant du régime qui tam (c'est-à-dire recevant un pourcentage des sommes ainsi économisées) : voir Dyck A., A. Morse et L. Zingales (2010) : « Who Blows the Whistle on Corporate Fraud? ॥, Journal of Finance, vol. 65, n 6, pp. 2213-2253. Nous ne voulons évidemment pas affirmer que les deux situations sont similaires, mais le cas du whistleblowing montre bien que les incitations à participer au processus doivent être pensées.

${ }^{30}$ À l'instar de l'Organisme national de l'eau et des milieux aquatiques (ONEMA) qui collecte et met en ligne des éléments d'information sur le coût, la qualité et les caractéristiques des services publics de l'eau, qu'ils soient en régie publique ou en délégation de service public même si la collecte reste incomplète en l'absence d'obligation et de sanction.
} 


\section{Renforcer les compétences de la partie publique}

\section{La professionnalisation des acheteurs publics}

Dans la plupart des pays de l'OCDE, le métier d'acheteur public ne bénéficie d'aucune reconnaissance spécifique. En France, il n'est référencé dans le Répertoire interministériel des métiers que depuis 2010 . On a longtemps reproché aux administrations de confier la gestion de leurs marchés publics à des personnes ayant reçu une formation uniquement juridique, indispensable pour que les procédures soient conformes à la législation mais insuffisantes pour réellement optimiser la commande publique. C'est moins vrai aujourd'hui : une récente étude de l'Union des groupements d'achat public (UGAP) montre en effet que $63 \%$ des acheteurs publics n'ont pas de profil juridique ${ }^{31}$. Toutefois, cette même étude indique que $61 \%$ des acheteurs publics ont intégré les services achats après une mobilité interne, sans expérience préalable en la matière, et que seulement $39 \%$ ont suivi une formation au métier d'acheteur. Enfin, l'étude indique que plus des deux tiers des acheteurs reconnaissent ne pas avoir une bonne connaissance du tissu économique et industriel et près de la moitié d'entre eux affirme ne faire aucune veille économique et technologique. Le renforcement de la professionnalisation de ces fonctions et la responsabilisation des acheteurs/responsables de la commande publique sont ainsi des leviers pour rationaliser la commande. En parallèle, offrir aux agents la possibilité de progresser et d'acquérir de nouvelles compétences grâce à des actions de formation peut faciliter la réduction du turnover et ainsi l'accumulation d'expérience et la « mémoire » des contrats.

La professionnalisation doit spécialement être renforcée, pour les contrats plus complexes de type concessions et contrats de partenariat, sur le suivi ex post des contrats. II est en effet nécessaire de comprendre et de contrôler l'information fournie par les délégataires et d'avoir une vision globale des enjeux à la fois économiques et techniques des projets. Cela doit faciliter l'adaptabilité des contrats (les renégociations indispensables pour des contrats de long terme) tout en renforçant le pouvoir de négociation de la partie publique ${ }^{32}$.

\section{Recommandation 8. Renforcer la professionnalisation et les compétences des acheteurs publics et des gestionnaires de projets. Stimuler l'attractivité de ces métiers par des possibilités d'évolution professionnelle.}

\section{Centraliser les achats autant que possible et capitaliser l'expérience}

La centralisation de la fonction achat (notamment dans le cadre des marchés publics de services et de fournitures mais aussi, dans une moindre mesure, de travaux) peut engendrer des économies substantielles. En effet, la centralisation permet de réaliser des économies d'échelle en capitalisant et en développant les bonnes pratiques, en mutualisant les procédures (diminution des coûts de fonctionnement et de passation) et en facilitant les groupements de commandes. L'exemple de la Corée du Sud est intéressant. Sur les 85 milliards d'euros d'achat public en 2013, le PPS coréen en a géré plus de 28 milliards. Le PPS poursuit ainsi, en regroupant les commandes publiques et en gérant les appels d'offres, des objectifs d'efficacité économique (économies d'échelle, transparence, simplification, concurrence). II poursuit aussi des objectifs en termes de montée en compétences des administrations publiques (formation, retour d'expériences...) et des entreprises coréennes (formation et aide afin de réussir sur les marchés étrangers). Ainsi, plus de $66 \%$ du montant total des marchés publics géré par le PPS est allé à des PME en 2013 (49\% pour les marchés de travaux).

En France, I'UGAP, centrale d'achat publique placée sous la double tutelle du ministère de l'Économie, des Finances et de l'Industrie et du ministère de l'Éducation nationale, est la seule centrale d'achat public généraliste. Son poids est limité puisqu'elle ne passe que pour 2 milliards d'euros de commandes par an.

Une réflexion doit être menée pour déterminer à quel niveau la centralisation des achats serait pertinente. La puissance publique pourrait ainsi organiser la mutualisation des ressources et des compétences au sein de centres de services partagés, pilotés par les régions ou les intercommunalités. L'existence de ces services mutualisés permettrait aux petites collectivités et établissements publics (Chambres de commerce, préfectures, tribunaux, hôpitaux, etc.), n'ayant pas nécessairement les moyens de recruter des acheteurs dédiés, de professionnaliser leurs achats ${ }^{33}$.

Toutefois, la question de la centralisation est complexe. D'un côté, la centralisation est porteuse de gains d'efficacité importants (retours d'expériences plus nombreux/spécialisation des personnels, professionnalisme et, enfin, pouvoir de négociation supérieur). De l'autre, elle déresponsabilise et peut finalement conduire, comme nombre de plaintes quant aux achats centralisés l'attestent, à des prix plus élevés (le décideur n'est qu'en partie le payeur), une lenteur d'exécution ou une offre limitée et peu flexible face à des besoins spécifiques.

\footnotetext{
${ }^{31}$ Enquête réalisée par I'UGAP et le magazine Décision Achats au cours du deuxième trimestre 2011, à laquelle 370 responsables d'achat ont répondu.

${ }^{32}$ En matière de délégations de service public, la plupart des collectivités font appel à des cabinets spécialisés (assistants maîtres d'ouvrage) pour les aider dans la préparation de l'appel d'offres et l'analyse des réponses des candidats.

${ }^{33}$ Le groupement de commandes pour les services de communications électroniques par le Syndicat intercommunal de la périphérie de Paris (SIPPEREC) est un bon exemple.
} 
Devant le problème lié à la déresponsabilisation, on peut imaginer différentes solutions, aucune d'entre elles n'étant parfaite : une clause d'《opt-out » - cette dernière protège les échelons inférieurs contre le manque de réactivité mais elle réduit le gain d'efficacité et de professionnalisme du donneur d'ordres ; une concurrence entre plusieurs acheteurs centralisés cherchant à obtenir une « part de marché » en redoublant d'efficacité. Un danger avec cette dernière solution étant le risque de choix plus politiques qu'économiques : il conviendrait alors de bien veiller à l'indépendance des centrales d'achats non seulement vis-à-vis des entreprises sous-traitantes, mais aussi vis-à-vis des donneurs d'ordres.

Au total, nous pensons que la centralisation des achats doit être développée davantage, spécialement pour les biens et services standards, mais qu'elle doit rester une possibilité offerte aux petits pouvoirs adjudicateurs et non un système obligatoire qui risquerait d'engendrer une mauvaise prise en compte des besoins, un allongement des délais, une diminution des choix ou encore une possibilité réduite de contracter avec des acteurs locaux.

\section{Recommandation 9. Centraliser autant que possible les achats pour les biens et services standardisés ; créer une concurrence entre centrales d'achat complètement indépendantes et professionnelles ; laisser la possibilité aux acheteurs publics de décentraliser leurs achats pour ne pas perdre en flexibilité lorsque celle-ci s'avère utile.}

\section{Renforcer l'encadrement pour les marchés les plus importants}

Pour s'assurer que les trois piliers " transparence ", " concurrence " et " compétences ॥ accroissent l'efficacité de la commande publique, les recommandations précédemment avancées doivent s'accompagner d'un plus grand encadrement en amont comme en aval de l'achat public pour les contrats les plus importants, pour lesquels un contrôle plus coûteux se justifie. En amont, il est nécessaire d'avoir systématiquement recours à une évaluation préalable : coût complet et avantages anticipés pour chaque projet selon l'outil de commande publique choisi. Ces évaluations préalables, limitées aux projets dépassant un certain seuil, permettront de déterminer la forme contractuelle la plus adaptée pour répondre à la demande publique, de mieux profiler le contrat et de mieux appréhender le coût global du marché considéré ${ }^{34}$.

Cet encadrement doit être étendu en aval par un suivi des renégociations. Outre la responsabilisation des acteurs, cet encadrement permettra de confronter les conditions d'exécution des marchés aux conclusions des études menées lors de la phase précontractuelle et ayant conduit à choisir tel mode d'organisation plutôt que tel autre. L'ensemble de ces évaluations doit être mené par une agence, ce qui par ailleurs faciliterait la centralisation des données et des résultats et permettrait de comparer les meilleures pratiques contractuelles.

Recommandation 10. Confier à une agence l'évaluation en amont et en aval de tous les outils de la commande publique dès lors que les montants dépassent un seuil, par exemple 50 millions d'euros.

Ce seuil devra être fixé de manière réaliste en fonction des moyens confiés à l'agence d'évaluation afin de ne pas ralentir les projets d'investissements publics.

\section{Conclusion}

La liberté accrue qui va être laissée aux pouvoirs adjudicateurs français, notamment dans la phase de sélection mais aussi dans la phase d'exécution des contrats, est potentiellement bénéfique à condition que cette liberté s'inscrive dans le cadre d'une transparence renforcée, d'une concurrence effective et du développement de compétences spécifiques.

\footnotetext{
${ }^{34}$ En application du Code général des collectivités territoriales, les assemblées délibérantes doivent se prononcer sur le principe de chaque délégation de service public. Les collectivités sont donc tenues d'étudier et de comparer les coûts, les avantages et les inconvénients d'une exploitation en régie à ceux d'une exploitation en gestion déléguée. Les études conduites doivent être impartiales et exhaustives. Les conditions de mise en œuvre de ces dispositions sont cependant variables, comme le note régulièrement la Cour des comptes.
}

\section{conseil d'analyse économique}

Le Conseil d'analyse économique, créé auprès du Premier ministre, a pour mission d'éclairer, par la confrontation des points de vue et des analyses de ses membres, les choix du Gouvernement en matière économique.

Membres Agnès Bénassy-Quéré, Antoine Bozio, Pierre Cahuc, Brigitte Dormont, Lionel Fontagné, Cecilia García-Peñalosa, Philippe Martin, Pierre Mohnen, Xavier Ragot, Jean Tirole, Alain Trannoy, Étienne Wasmer, Guntram Wolff

Conseillers scientifiques

Jean Beuve, Clément Carbonnier,

Jézabel Couppey-Soubeyran,

Manon Domingues Dos Santos

Assistante de recherche

Alice Keogh
Correspondante

Anne Perrot
Les Notes du Conseil d'analyse économique ISSN 2273-8525

Directrice de la publication Agnès Bénassy-Quéré Rédactrice en chef Hélène Paris

Réalisation Christine Carl

Contact Presse Christine Carl

christine.carl@cae-eco.fr Tél. : 0142757747 